\title{
La teoría de la justicia de Michael Walzer frente al pluralismo y la desigualdad educativa en México
}

José Aguilar Sahagún

Instituto de Formación Filosófica Intercongregacional, México

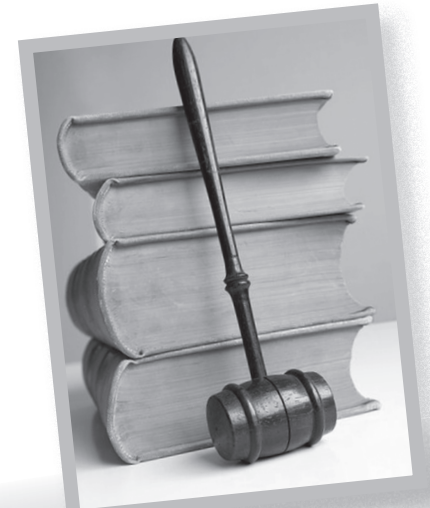

RESUMEN: El filósofo estadounidense Michael Walzer propone una concepción de la justicia en función de una diferenciación de los bienes sociales. Al ámbito de la educación corresponden criterios peculiares de justicia. Las ideas de este pensador resultan de particular interés, dado que su propuesta teórica está estrechamente ligada al contexto cultural y a la observación empírica. Aquí se reflexiona sobre su propuesta a la luz de los problemas educativos en el país de origen del autor.

ABSTRACT: The American philosopher Michael Walzer poses a conception of justice regarding a differentiation on social goods. The educational milieu has peculiar criteria on justice. Walzer's ideas are particularly interesting, for his theoretical proposal is closely linked to the cultural context and the empirical observation. The author reflects on Walzer's proposal considering the educational problems in his country. 
a educación es un bien social de una naturaleza peculiar. Las dificultades de una teoría de la justicia educativa se desprenden de esa constatación, como lo muestra el análisis de Latapí. El filósofo estadounidense Michael Walzer ${ }^{1}$ (19352002) propone una concepción de la justicia en función de una diferenciación de los bienes sociales. Al ámbito de la educación corresponden criterios peculiares de justicia.. Con su obra Esferas de la justicia ${ }^{2}$ (1983) Walzer ha abierto un horizonte de reflexión que ha fecundado la discusión sobre este concepto en el contexto del Ilamado debate liberalismo-comunitarismo ${ }^{3}$. El planteamiento de Walzer es de particular interés, dado que su propuesta teórica está estrechamente ligada al contexto cultural y a la observación empírica. Los planteamientos de Walzer se ubican conscientemente en la realidad político social de los
EE.UU., pero han venido cobrando un fuerte eco en otras latitudes, como es el mundo académico de Europa central ${ }^{4}$. El presente ensa$y^{5}{ }^{5}$ ofrece una presentación de su propuesta, y examina sus alcances a la luz de los graves problemas de la desigualdad educativa en México. Este estudio consta de cinco partes. En la primera se presenta la concepción de Walzer sobre los bienes sociales para, en la segunda parte, analizar su concepción de la educación como bien social. En la tercera parte se presenta el marco general de la teoría de Walzer sobre la igualdad compleja. En la cuarta parte se ofrecen elementos para un balance de su pensamiento, tanto desde un punto de vista teórico general como en la perspectiva de la problemática de la desigualdad educativa en México. En la quinta parte se exponen algunas conclusiones.

\section{Una teoría de los bienes sociales}

$\mathrm{P}$ ara explicar y delimitar los límites del pluralismo de las posibilidades de distribución Walzer considera inprescindible una teoría sobre los bienes. "La justicia requiere defender la diferencia -bienes diferentes distribuidos por razones diferentes a grupos de gente diferentes..." (Walzer: 1996: 65)

En la opinión de Walzer, los bienes no tienen una naturaleza esencial y, en consecuencia, la justicia distributiva debe tener una relación con el lugar que esos bienes ocupan en la vida (mental y material) de la gente entre quienes se distribuyen. La máxima es muy simple en su formulación: "la justicia distributiva es relativa a los significados sociales." (Walzer: 1996: 58).

Para empezar, queda descartada la idea de que existe un conjunto de bienes universales abstractos, objeto de la distribución. En lugar de partir de la idea común de acuerdo con la cual la justicia distributiva es el pro- 
ceso social en el que un conjunto de bienes pasa de unas personas a otras, Walzer propone tomar como punto de partida la concepción y creación de los bienes mismos que los grupos distribuyen entre sí. Los bienes no son entes abstractos que han de ser asignados de acuerdo con ciertos principios generales. Los bienes son indisociables de los significados que la gente les atribuye, y que constituyen el medio por excelencia de relaciones sociales.

Si insistimos en la diferenciación y en la especificidad en el ámbito de las pretensiones, la suma de nuestros rechazos, reconocimientos y cualificaciones es lo que llamo "igualdad compleja": es decir, la condición social en la que ningún grupo particular domina los diferentes procesos distributivos. (Walzer 1996: 65).

Para Walzer es más importante el tipo de bienes por distribuir que su cantidad. La desigualdad es ilegítima cuando es opresora o "tiránica", es decir, cuando un tipo de bien, como por ejemplo, el dinero, domina o influye sobre los otros bienes. La distribución desigual de un bien es legítima dentro de su propia esfera de influencia. Pero un tipo de bien no debería influir sobre otras esferas. Se pueden comprar muchas cosas con el dinero, pero no bienes como la salud o el poder político, cuya distribución obedece a otras reglas, que están en función de su significado social. La desigualdad de riqueza se justifica en la medida en que no ejerce influjo sobre ámbitos que no deberían poder ser comprados con dinero. En esto consiste la igualdad compleja.

\section{La educación como bien social}

punto de partida del análisis de Walzer es el pluralismo en la concepción de los bienes. $\mathrm{Ni}$ siquiera la constitución de la sociedad que, de acuerdo con Aristóteles, es lo que ofrecería la base para llegar a acuerdos sobre aspectos particulares de la vida pública, puede ser considerada como objeto de un acuerdo dado de una vez por todas.

La educación no es un bien cuyo significado sea completamente relativo. Tampoco podemos derivar de él los criterios necesarios para determinar la función que debería desempeñar dentro de una sociedad o los efectos que serían deseables dentro de ella. La función social de la educación es muy variable. No se puede afirmar, por ejemplo, que la función de las escuelas se reduzca a reproducir el status quo de una sociedad: sus jerarquías, su ideología dominante, su división de trabajo, etc. De ser así, carecería de sentido 
plantear la cuestión de la justa distribución de los bienes educativos: escuelas, maestros, ideas. Lo decisivo es que este conjunto de bienes tiene un carácter específico que ha de ser distribuido en correspondencia con un conjunto de procesos distributivos de otros bienes sociales que generan sus propias esferas de equidad, independientes de los que funcionan para la distribución de otros bienes.

Para Walzer la educación es un bien social que en cierto modo "distribuye" a las personas no sólo el tipo de futuro que puedan Ilegar a vivir, sino también su realidad presente, dentro del conjunto de condiciones históricas que inciden en los procesos distributivos de todos los bienes. El proceso educativo adopta una estructura normativa peculiar. A Walzer le interesa subrayar que la manera en que cada uno de los bienes educativos se tenga que distribuir depende de lo que la comunidad vaya decidiendo durante el proceso histórico en el que se crean los significados sociales. (1983: p. 198).

Sobre la base de esos consensos es posible decidir, por ejemplo, qué niños y niñas se han de admitir en la escuela, qué tipo de escuela habrán de visitar, qué criterios de admisión se habrán de aplicar, qué tipo de estudios o alternativas se les van a ofrecer, la duración del período escolar, etc.

\subsection{Educación básica}

La educación básica ha de distribuirse de acuerdo con los dos criterios válidos para todas las esferas de la justicia: autonomía y equidad. Por su propia naturaleza, las escuelas no pueden ser excluyentes. Cuando lo son, es porque son confiscadas por una élite social. "Forma parte del honor de los maestros — afirma Walzer- atender las necesidades educativas sin consideración a la clase social o al ingreso de quienes asisten a la escuela" (1983: p. 202). En este sentido, Walzer está de acuerdo con Aristóteles, para quien, en oposición a las prácticas comunes de la Atenas de su época, consideraba que el sistema educativo de la comunidad política debe ser el mismo para todos, y que su atención ha de ser considerada como un asunto público.

Walzer concibe la igualdad educativa como una forma de prestación en la que todos los niños y las niñas son considerados como futuros ciudadanos con las mismas necesidades de conocimiento. EI ideal de participación (Membership) se alcanza si a todos se les enseña lo mismo. No es admisible que el tipo de educación que recibe un niño o una niña dependa de la posición social o de la capacidad económica de sus padres.

En contraste con esta comprensión de la igualdad compleja, la igualdad simple considera la necesidad como único criterio de distribución, en este caso, la necesidad que tienen todos los futuros ciudadanos de recibir una educación. Si, en cambio, se considera la educación 
desde el interior de la escuela, es posible constatar que además de la necesidad, es preciso considerar otros criterios en la distribución del conocimiento. El interés, el empeño o la capacidad de los escolares son criterios por lo menos tan relevantes como el de la necesidad ${ }^{6}$.

La justicia compleja no busca suprimir todas las diferencias sociales que surgen de forma natural dentro de una sociedad plural. Lo que Walzer propone es postergar las desigualdades, de tal manera que, ante todo, los niños y las niñas aprendan a ser ciudadanos $y$, en segundo término, a desempeñar cualquier oficio, incluyendo a los servicios profesionales. Hay materias que todos los niños y las niñas deben conocer para poder ser ciudadanos y ciudadanas. Si el acento se pone en este aspecto, la escuela deja de ser el monopolio elitista para unos cuantos, en el que automáticamente se decide el lugar social y el oficio que han de aprender los futuros ciudadanos. No existen vías privilegiadas de acceso a la ciudadanía. El mejor desempeño escolar no ha de ser considerado como la manera más corta de llegar a ser ciudadano, ni la escuela como garantía de privilegios. Su tarea es la de ofrecer el bagaje común que sustenta la vida política y social ${ }^{7}$.

Bajo el supuesto de un mismo interés y una capacidad promedio, el criterio de justicia con respecto a la educación básica es el de la necesidad. A este nivel, Walzer aplica el criterio de equidad desde el punto de vista de la igualdad simple, frente al que opone su teoría de una igualdad compleja. Lo decisivo es la necesidad que tiene cada niño o niña de crecer dentro de una comunidad democrática y de ocupar su lugar dentro de ella como un ciudadano competente. Este es el fin principal de las escuelas. De ahí que éstas deberían funcionar de acuerdo con formas de participación que en cierto modo anticipen los patrones de la vida democrática de los adultos (1983: p. 217).

Siguiendo al filósofo estadounidense John Dewey (1961) Walzer considera que la educación ha de contribuir a la reproducción de la democracia, entendida ante todo como forma de vida, cuyo último fin consiste en la formación para la participación. Este tipo de educación supone que cada persona es capaz de autodeterminarse en el proceso de vida de la comunidad de la que forma parte, dentro del marco de una sociedad estructurada democráticamente.

El relativismo cultural de Walzer oscila entre la afirmación de un fin educativo fundamental a cuyo servicio han de ponerse todos los demás bienes - la formación de ciudadanos y ciudadanas-, y el énfasis en la pluralidad de significados que éstos cobran dentro de cada cultura ${ }^{8}$.

Desde la perspectiva de la igualdad simple, el punto de partida de la educación democrática es el principio según el cual para obtener un mismo fin se ha de emplear 
y exigir el mismo trabajo. Dentro de este esquema la educación se imparte $-y$, en este sentido, se distribuye- a todos los niños y niñas, y se les ofrece la ayuda necesaria para que dominen el mismo tipo de conocimientos.

Para Walzer esto tiene el efecto de "robar a la economía su único proletariado legítimo, el de los jóvenes, y el hacer más difícil de lo necesario el acceso del proletariado real a rangos superiores" (1983: 206). No parece que en este punto Walzer se refiera a la educación básica, sino a las fases ulteriores de educación media y superior.

Si bien la prolongación del tiempo que un estudiante puede dedicar para su educación constituye un avance de gran importancia en la búsqueda de la igualdad, Walzer considera que, en muchos casos, esto deja de ser verdad, dado que no es posible que un mismo estilo de vida sea el adecuado para todos los estudiantes. La única extensión legítima de la cobertura en educación básica en correspondencia con una sociedad democrática es la oferta de oportunidades iguales reales y de auténtica libertad de pensamiento para todos los niños y niñas, y no sólo para unos cuantos, seleccionados de acuerdo con criterios puramente convencionales. En este punto, Walzer establece un límite al relativismo cultural. A nivel de educación básica, el valor de la igualdad democrática tiene preeminencia sobre todos los valores que de alguna manera puedan ponerla en peligro.
De modo consecuente, Walzer limita el alcance de su propia teoría. Todos los ciudadanos han de poder dar su punto de vista y someter a juicio los criterios de una teoría de la justicia educativa. Si la determinación de estos criterios se dejara en manos de un grupo de expertos seleccionados por el Estado se faltaría a una exigencia elemental de la justicia democrática9 ${ }^{9}$.

Tanto las limitaciones en el presupuesto para la educación como en el número de puestos que supongan cierto tipo de formación no deben constituir un obstáculo insuperable para que los interesados logren con la máxima eficiencia el tipo de aprendizaje que necesitan. La educación de ciudadanos constituye una garantía de bienestar social. Por eso es imprescindible ofrecer la misma educación elemental a todos los niños y niñas.

La formación de especialistas en alguna área del conocimiento - considerados como un subconjunto de ciudadanos dentro del gran conjunto constituido por toda la ciudadanía- no es menos importante, y ha de ser objeto de una selección ulterior, que no puede estar sujeta a criterios convencionales o arbitrarios.

Así, la búsqueda de estatus, de poder o de riqueza que, normalmente, están asociados a cierta posición profesional, provocaría graves consecuencias morales dentro de una sociedad si quienes aspiran a esos beneficios no se esforzaran por 
obtener una buena educación, es decir, una educación que los capacite para ser, ante todo, ciudadanos, al margen de lo que esos beneficios significan para ellos. Esta apreciación pone de manifiesto el realismo de Walzer. La educación como un bien o un conjunto de bienes, tiene la peculiaridad de potenciar por sí misma el acceso a otros bienes, en detrimento de su fin básico, que es al mismo tiempo el fundamento de la democracia, de la igualdad y del bienestar social: la formación de ciudadanos (1983: p. 211).

Es lamentable que las observaciones de Walzer sobre la educación media y superior sean muy escuetas. En Esferas de la justicia, Walzer se limita a constatar que, si se concibe la educación media y superior como una empresa comercial, automáticamente se la convierte en un instrumento de tiranía en manos de quienes la orienten en este sentido. El mercado nunca puede constituir una esfera completamente cerrada en la que lo único que cuenta es el dinero (1983: p. 213). Cabe decir que la utilización de las instituciones educativas con fines ideológicos es otra manera de ejercer un tipo de tiranía sobre la educación.

Las escuelas nunca pueden actuar con plena autonomía. Lo decisivo es, en todo caso, que si verdaderamente se busca que gocen de algún grado de autonomía, es imprescindible que se establezcan medidas que impidan que los criterios válidos en otras esferas distribu- tivas invadan la esfera de los bienes educativos (Idem, p. 213).

\subsection{Compensación de las desigualdades educativas}

Las diferencias entre escuelas públicas y privadas es un hecho del que hay que partir, dado que su existencia forma parte de la manera en que una comunidad ha creado el bien social de la educación. Walzer parece percibir que las escuelas privadas favorecen de suyo la desigualdad social, si es que no son expresión de la misma. Pero, de forma consistente, su interés se centra en subrayar que los criterios de la igualdad educativa se generan a partir del conjunto de los significados convencionales de los bienes que constituyen al ámbito de la educación en una esfera cuya propia autonomía es preciso respetar. A fin de lograrlo, Walzer propone un mecanismo de compensación que contribuya a crear condiciones de mayor igualdad educativa, que consiste en la creación de un plan de títulos de pago por medio de los cuales los padres de familia reciben una parte de los impuestos de la ciudadanía, con el fin de que dispongan de recursos adicionales para solventar los gastos que genera la educación de los hijos o de las hijas. Es importante evitar que se establezca un sistema educativo discriminatorio entre diferentes tipos de estudiantes dentro de las mismas escuelas ("a two class system", Ídem, p. 221).

Si bien para Walzer es claro que el fin de la educación escolarizada 
es la integración social de futuros ciudadanos, no resulta fácil precisar el tipo de medios necesarios para alcanzar ese objetivo. La dinámica social tiende a ejercer presión en el sentido de configurar un sistema dentro del cual la composición social de las escuelas tiende a la nivelación de la educación de acuerdo con el principio de asociación proporcional: diferentes tipos de niños y de niñas se mezclan en la misma proporción en todas las escuelas dentro de un área determinada. La proporción varía de un área a otra en función del carácter general de la población estudiantil. La aplicación de este tipo de criterio tiene el efecto de descartar los mecanismos de refuerzo que permitan que todos los niños y las niñas reciban los mismos conocimientos básicos que les permitirán llegar a ejercer su ciudadanía. El resultado de este tipo de procedimientos es la formación para una ciudadanía ideológica, y no para una ciudadanía que parte de la realidad, es decir, igualitaria y pluralista (1983: p. 223).

\section{Educación e igualdad compleja}

$\mathrm{T}$ oda idea de justicia parte de una manera particular de concebir la igualdad. Walzer concibe la igualdad como una igualdad compleja, en contraposición con la idea de igualdad que él califica como simple. Para comprender esta distinción es necesario tener presente que para él, el valor de la igualdad no puede desligarse del pluralismo, tanto de bienes como de criterios de distribución. La igualdad simple supone la existencia de un único criterio universal de distribución aplicable indistintamente a todo tipo de bienes. En contraste, la igualdad compleja toma en cuenta la diversidad de bienes sociales que se intercambian dentro de una sociedad, cada uno de los cuales constituye de por sí una esfera independiente dentro de la cual se establecen los criterios normativos de distribución.
Los múltiples bienes colectivos que se intercambian, producen y consumen dentro de una sociedad y a los que el valor de la justicia intenta ordenar, están asociados a una diversidad de procesos, agentes y criterios de distribución (Walzer: 1983, p.3).

Para lograr una idea adecuada de la justicia distributiva es necesario considerar tanto la diversidad de los bienes, como de los criterios de distribución asociados a ellos en cada sociedad. Walzer toma como punto de partida el carácter particular, histórico y la pertenencia a una comunidad concreta de quienes buscan criterios de justicia adecuados a determinados bienes. La cuestión de fondo en relación con la justicia se refiere a la elección que harían personas concretas que, dentro de su contexto cultural, se han propuesto compartir sus bie- 
nes. "La autonomía es un principio distributivo básico que se deriva de la propia diferenciación de bienes." (Walzer 1996: 64).

Como construcción humana, la pregunta por la justicia puede plantearse de muchas maneras, y da lugar a una amplia gama de elecciones políticas, porque siempre ha de tomar en cuenta las opciones que determinados grupos ya han realizado a lo largo de su historia común a partir de la comprensión compartida de determinados valores y bienes.
La tesis que Walzer intenta sustentar es que en su misma estructura, los principios de justicia son plurales; la distribución de los bienes sociales ha de obedecer a diversos criterios. Y que, en el fondo, todas estas diferencias se derivan de una comprensión distinta de los bienes sociales mismos, resultado inevitable del proceso particular, histórico y cultural en que están insertos los sujetos, vinculados a sus respectivas comunidades.

\section{Igualdad compleja y educación: Un balance}

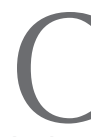

on el fin de hacer una ponderación adecuada de la propuesta de Walzer, el siguiente balance está articulado en tres partes. En un primer momento, centraré mis reflexiones sobre el alcance y los límites de la teoría de Walzer a nivel general. En un segundo momento, haré algunas observaciones críticas que ponen de relieve algunos puntos inconsistentes de la idea de igualdad compleja. Finalmente, trataré de ver en qué medida su teoría ofrece elementos para comprender de manera adecuada los problemas de la desigualdad educativa en México, y de mostrar algunas condiciones bajo las cuales serían aplicables en el contexto mexicano algunas de las intuiciones centrales de su teoría ${ }^{10}$.

\subsection{Apreciación general de la propuesta}

Para Walzer, el problema central de la justicia distributiva en materia de educación consiste en crear las condiciones bajo las cuales los escolares (Walzer habla explícitamente de niños y niñas) tengan acceso a un aprendizaje común sin que esto signifique lo que cada uno de ellos tiene de más particular, por ejemplo, desde el punto de vista genético o social. El énfasis está puesto tanto en la igualdad como en el pluralismo. La teoría de la igualdad compleja ofrece una solución a este problema, en el que es necesario atender al modelo escolar normativo que se desprende de su teoría de los bienes sociales y del valor del pluralismo, por un lado, y a las exigencias igualitarias del régimen de vida dentro de una democracia, por el otro. La teoría no ofrece una única solución, dado que entre las instituciones educativas y las instancias de la vida 
política existen (o deberían existir) instituciones que tienen la función de mediación, cuya naturaleza sólo puede determinarse con relación a las fuerzas sociales que representan ambas partes dentro de cada marco cultural específico (Walzer: 1983, p. 216).

\section{2 ¿"Desigualdad compleja"?}

Dentro la teoría distributiva de Walzer no hay lugar para la noción del bien común ${ }^{11}$. Podríamos decir que para este pensador el bien común consiste en una sociedad plural dentro de la cual una ciudadanía activa respeta las reglas que, dentro de cada comunidad, se desprenden de los significados sociales que les han atribuido a los bienes.

La idea de que los criterios de justicia distributiva se desprenden de los significados sociales que les atribuye una comunidad tiene un punto a su favor. Cuando ciertos bienes están al alcance de quienes los necesitan, la desigualdad de riqueza tiene un impacto social menos relevante. Muchos bienes tienen un enorme valor de uso individual sólo por el valor simbólico que se les atribuye. En esos casos, la desigualdad de su distribución carece de importancia (Walzer: 1983, p. 108).

Sin embargo, se puede constatar que la separación de esferas que Walzer establece en su teoría es hasta cierto punto artificial. En realidad, el dinero tiene un efecto directo sobre el acceso a la educación, al poder político, a la atención médica, a la cultura, etc. Es poco realista sostener, por ejemplo, la legitimidad de enormes desigualdades en el ingreso y la acumulación de riqueza, y prohibir al mismo tiempo el influjo entre las distintas esferas de bienes. El problema de fondo parece radicar en que Walzer no toma en consideración que de hecho las esferas se sobreponen unas a otras. En este sentido, su teoría puede ser caracterizada más bien como una "desigualdad compleja", porque en ella no se plantea la exigencia de que algunos bienes deban ser igualmente distribuidos. Este modelo de igualdad es muy limitado frente a las exigencias de la realidad. (DeMarco: 1996, p. 226).

Cabe destacar que Walzer no descarta del todo la idea de lo que él caracteriza como "igualdad simple y directa", en oposición a la igualdad compleja, que es diferenciada y específica. La idea de igualdad simple puede servir como un punto de referencia crítico frente a graves injusticias. Su límite es su incapacidad de ser aplicada en todos los ámbitos de los bienes a distribuir (Walzer 1996: 65). Y es que "cualquier intento por reforzar la igualdad más allá de ciertos límites se hace eo ipso autocontradictorio, porque requiere una concentración radical de poder político y por tanto de una radical desigualdad de distribución" (Ídem., passim).

Cabe preguntar si esta apreciación es válida respecto de las políticas educativas que el gobierno y la sociedad tienen que aplicar para 
crear una mayor "igualdad de oportunidades educativas".

\subsection{La igualdad compleja y la esfera de la educación en México}

Walzer ha puesto en el centro de sus reflexiones la exigencia de diferenciación de ámbitos en la aplicación de cualquier principio normativo. Su pensamiento manifiesta una señalada opción por un estilo de pensamiento normativo "débil" en el sentido de que, decididamente, deja atrás la idea de unos cuantos principios universales válidos para todos los ámbitos sociales y contextos culturales. En clara oposición a lo que a sus ojos se presenta como un universalismo abstracto - como el que representa el pensamiento liberal del primer Rawls ${ }^{12}$-, Walzer afirma el valor del pluralismo, se podría decir, por respeto a la realidad misma.

Al poner de relieve la vinculación de criterios normativos a los significados de los bienes sociales, Walzer destaca un criterio que de hecho tiende a normar las decisiones que se toman dentro de determinados ámbitos. Esto es en cierto modo una constatación, no una norma. Cabría preguntar: ¿En qué contexto social las decisiones en materia de distribución de bienes no están determinadas por el significado que éstos tienen para los miembros de la comunidad afectada? La pregunta no es trivial, pero su respuesta se vuelve problemática en contextos culturales amplios, en donde el concepto de comunidad no es necesariamente el resultado de consensos básicos, sino el producto de una ideología dominante, por ejemplo, de tipo nacionalista. La cuestión se vuelve más aguda cuanto menos homogénea es una comunidad y cuanto menor es la participación de sus miembros en la creación de los significados sociales de los bienes que se distribuyen dentro de ella.

El acento en el pluralismo contribuye a encarnar cualquier criterio normativo que tenga la pretensión de alcance universal, al margen de las personas y de las culturas concretas en las que podrían tener validez. En el caso mexicano, aún no se ha llegado al pleno reconocimiento de la pluralidad cultural y étnica ni se han sacado todas las consecuencias que esto representa para cualquier teoría de carácter normativo. Los movimientos sociales que reivindican el pluralismo ya están en marcha, y no es previsible el alcance de la dinámica social que han venido cobrando. En este sentido, una teoría de la justicia en correspondencia con nuestra realidad tendría que incorporar el principio de igualdad compleja.

Pero el reto es mucho mayor. El pluralismo es un valor dentro de una sociedad cuando no se le absolutiza. México se ha constituido ya, en menor o mayor medida, en una nación. Los mismos grupos que reivindican el derecho a la diferencia, se entienden a sí mismos como miembros de la nación en todo su 
sentido, es decir, como ciudadanos mexicanos $^{13}$.

El pluralismo ha de ir acompañado de criterios convergentes. El valor de la igualdad democrática enfatizado por Walzer supone la exigencia de la inclusión de todos los miembros de lo que de suyo constituye o podría constituir una "comunidad de comunidades". La posibilidad de constituir una "nación" de estas características depende de la capacidad de que todos los actores sociales sean en primer lugar sujetos, es decir, que se entiendan a sí mismos y actúen como seres autónomos, libres y responsables. Desde esta perspectiva, es hasta cierto punto secundario que la autocomprensión de los sujetos esté subordinada a identidades pre- valentemente colectivas. Lo decisivo es su capacidad de constituirse en verdaderos interlocutores, así como de que los significados que para ellos guardan los bienes sociales se conviertan en patrimonio común.

Lo anterior supone dos condiciones indispensables, que aún estamos lejos de alcanzar. Por parte del gobierno, la voluntad política del reconocimiento, es decir, hacer efectivo lo que ya está consignado en la Carta magna de nuestro país. Por parte de la sociedad en su conjunto, un proceso de maduración a través del cual se llegue, por una parte, a aceptar el valor de lo otro, sin absolutizarlo; y por otra, a valorarlo e integrarlo dentro del horizonte de significados de los bienes sociales que ya determinan su convivencia.

\section{Conclusiones}

1. La integración del valor del pluralismo dentro de una sociedad compleja, polarizada desde el punto de vista económico y sometida a procesos globales que abarcan desde la economía hasta los estratos más profundos de la cultura es una tarea urgente, de la que no pueden sustraerse ni el gobierno ni la sociedad civil. No parece que la teoría de Walzer, ni alguna otra teoría de la justicia, sean capaces de ofrecer criterios claros que respondan a estas exigencias. Porque lo que está en juego es el provecho que de ella puedan extraer todos los actores sociales.
2. Uno de los mayores retos que esto representa para la reflexión teórica normativa es sin duda la integración del factor tiempo. Los procesos de distribución y redistribución que verdaderamente estén a la altura de estas exigencias tienen que responder a las dinámicas de integración social, de consolidación de los significados sociales de los bienes y, particularmente, a la existencia de un conjunto de significados compartidos por todos los miembros de un país de las características del nuestro, como para que las políticas distributivas tengan un mínimo común denominador de 
alcance universal, al menos por lo que se refiere a los intereses nacionales ${ }^{14}$.

3. Otro reto para una teoría de estas características es la atención a las esferas concretas ya existentes dentro de cada comunidad, es decir, a los ámbitos cuyo orden de sentido está constituido por un conjunto de bienes a los que una comunidad ha asignado significados específicos. Si bien, en el caso de la descentralización educativa, las políticas orientadas a hacer real el principio de la federación pueden contribuir a hacer real este ideal, queda por dar solución a la problemática que plantea el intercambio de bienes educativos entre distintas comunidades de cada entidad federativa; los distintos niveles de exigencia educativa y académica, la diversidad de necesidades dentro de cada una de ellas, consideradas dentro del conjunto de un país de instituciones políticas débiles o corroídas por los males de la corrupción y con una economía poco estable y excluyente.

4. La compleja superposición de esferas que de hecho ocurre dentro de México hace muy difícil la aplicación del principio de igualdad compleja y el cumplimiento de las exigencias que trae consigo el reconocimiento del pluralismo. Por estas razones me parece que la propuesta de Michael Walzer no ofrece una solución adecuada al problema mayúsculo de la desigualdad educativa en México. En un contexto como el de nuestro país no es previsible, por lo menos en el corto plazo, la adopción y funcionamiento justo y eficaz de criterios armónicos en los procesos distributivos del conjunto de los bienes sociales. Las soluciones comenzarán a vislumbrarse en la medida en que la sociedad mexicana, en su diversidad y pluralidad, se constituya cada vez más en una sociedad civil con la vitalidad suficiente para devolver al Estado su función eminentemente subsidiaria.

5. No obstante, vale la pena tener en cuenta la llamada de atención sobre el riesgo permanente de que unas esferas ejerzan predominio e incluso tiranía sobre las otras. La teoría de Walzer sobre los bienes como conjunto de significados compartidos se presenta como una sugerente llamada de atención para evitar que en el ámbito de la educación intervengan otras esferas, ejerciendo un tipo de tiranía sobre ella, que no respeta al carácter específico de lo educativo que, de suyo, está constituido por un conjunto de bienes específicos, particularmente, el que se refiere al aprendizaje, la enseñanza y el desarrollo de las personas.

6. Concretamente, en el contexto mexicano cabría considerar la propuesta de evitar la tiranía de las esferas ajenas a la educación creando los mecanismos para crear condiciones de mayor igualdad educativa, como podría ser la creación de un plan de títulos de pago por medio de los cuales los padres de familia reciban una parte de los impuestos de la ciudadanía, que les 
permita disponer de recursos adicionales para solventar los gastos que genera la educación de sus hijos e hijas.

BiBLIOGRAFÍA

Bárcena, Fernando (1997), El oficio de la ciudadanía, Introducción a la educación política. Paidós, Barcelona.

DeMarco, Joseph P. (1996), Moral Theory, A Contemporary Overview. Jones and Bartlett Publishers, Boston y Londres.

Dewey, John (1961), Democracy and Education, Nueva York.

Krause, Skadi y Karsten Malowitz (1998), Michael Walzer, Zur Einführung. Junius, Hamburgo.

Latapí Pablo, "Reflexiones sobre la justicia en la educación" en Revista Latinoamericana de Estudios Educativos, Vol. XXIII, 1993, No. 2, México, pp. 12-22.

Morelia. . "Políticas educativas" en Tiempo educativo mexicano, Vol. II (1992),

Lebret, Louis Joseph, Suicidio o sobrevivencia de Occidente (1968). Descleé de Brower, Bilbao.

Rawls John (1971 1 ${ }^{\mathrm{a}}$ Ed.) A Theory of Justice. Harvard University Press, Harvard.

(1976) Teoría de la justicia. Fondo de Cultura Económica, México.

(1996) Liberalismo político. Fondo de Cultura Económica, México.

Walzer, Michael (1983), Spheres of Justice, A Defense of Pluralism and Equality. Basic Books, Nueva York.

(1996) Las esferas de la justicia, defensa del pluralismo y la igualdad. Fondo de Cultura Económica, México.

Thiebaut, Carlos (1998), Vindicación del ciudadano, Un sujeto reflexivo en una sociedad compleja. Paidós, Barcelona, Buenos Aires, México.

Notas

1 Michael Walzer es quizá el pensador más original en el debate entre liberales y comunitaristas. Walzer es un "intelectual político", inmerso en los conflictos concretos de la sociedad estadounidense y de algunos de los graves conflictos políticos mundiales. Ha sido profesor de la Universidad de Harvard y desde
1980 es profesor del Instituto de Estudios Avanzados de la Universidad de Princeton. Es editor de la revista Dissent, coeditor de la revista The New Republic, Political Theory y Philosopby and Public Affairs.

2 Las referencias a la obra de Walzer en este trabajo se basan en la edición original en inglés Spheres of Justice, A Defense of Pluralism and Equality (1983) Nueva York, y a la edición 
traducida al castellano (Fondo de Cultura Económica, México, 1996).

3 Este debate se refiere a la discusión entre teóricos y filósofos de lo político que se generó en los EE.UU. desde los años setenta, sobre todo a partir de la publicación de la teoría de la justicia (A Theory of Justice: 1971) del profesor de Harvard, John Rawls. Michael Walzer no se identifica con el conjunto e autores caracterizados como comunitarians (como Charles Taylor, Michael Sandel o Alastair McIntyre), aunque tiene puntos en común con ellos. Particularmente, su insistencia en los vínculos histórico culturales de las personas con sus respectivas comunidades y en su decidida opción por una izquierda liberal. Es ilustrativo consultar las obras de Carlos Thibeaut (1992), Los limites de la comunidad, Madrid y de Fernando Bárcena (1997) El oficio de la ciudadanía, Introducción a la educación política.

$4 \quad$ Ya existen numerosas publicaciones sobre el pensamiento de Walzer en Italia, España, Francia y Alemania, países en donde se han traducido sus obras. La recepción ha generado fecundos debates. Cf. Krause, Skadi y Malowitz Karsten (1998), con amplia bibliografía.

5 El presente trabajo forma parte del libro Educación, derechos de la infancia y derecho al desarrollo. (N. del E.)

6 Como ejemplo del significado social de la educación y de la estructura normativa que de ahí se sigue, Walzer señala que en la tradición judía la relación entre maestro y alumno se define particularmente sobre la base de los criterios señalados.
"Un mejor desempeño en la escuela no ofrece una vía privilegiada de acceso a la ciudadanía, ni un modo más rápido para llegar a ella. La educación escolar no es garantía de nada y se puede intercambiar por muy pocas cosas, pero provee el sustrato común de la vida política y social” (Walzer: 1983, p. 203).

8 El caso del Japón ilustra esta polaridad de forma eminente. Walzer observa que la igualdad educativa alcanzada en este país tuvo un desarrollo significativo por el hecho de que en las escuelas existía la consigna de no permitir la intromisión corporativista del gobierno. Sólo sobre esa base fue posible ofrecer auténtica educación común. Lo que ha contribuido a la igualdad educativa en este país es que las escuelas se unieron bajo una ideología común para resistir a la presión corporativista del gobierno oficial que, sometido a su vez a las presiones de la élite empresarial, se orientaba a la generación de desigualdades. Sólo sobre esta base fue posible ofrecer una auténtica educación común (1983: p. 204). En las escuelas que adoptaron esta ideología, el curriculum es exigente, adaptado a la capacidad de aprendizaje de los estudiantes que se ubican por encima del promedio. Esto constituye para Walzer un signo de escuelas sólidas y de maestros ambiciosos. La opinión común según la cual el fin de ofrecer educación a todos los posibles aspirantes conduce a una disminución en los niveles educativos es válida sólo con respecto a escuelas poco consolidadas, incapaces de ofrecer resistencia a la presión de una sociedad jerárquica. Otra característica de las escuelas fuertes es que en ellas no hay personal de mantenimiento. La comunidad edu- 


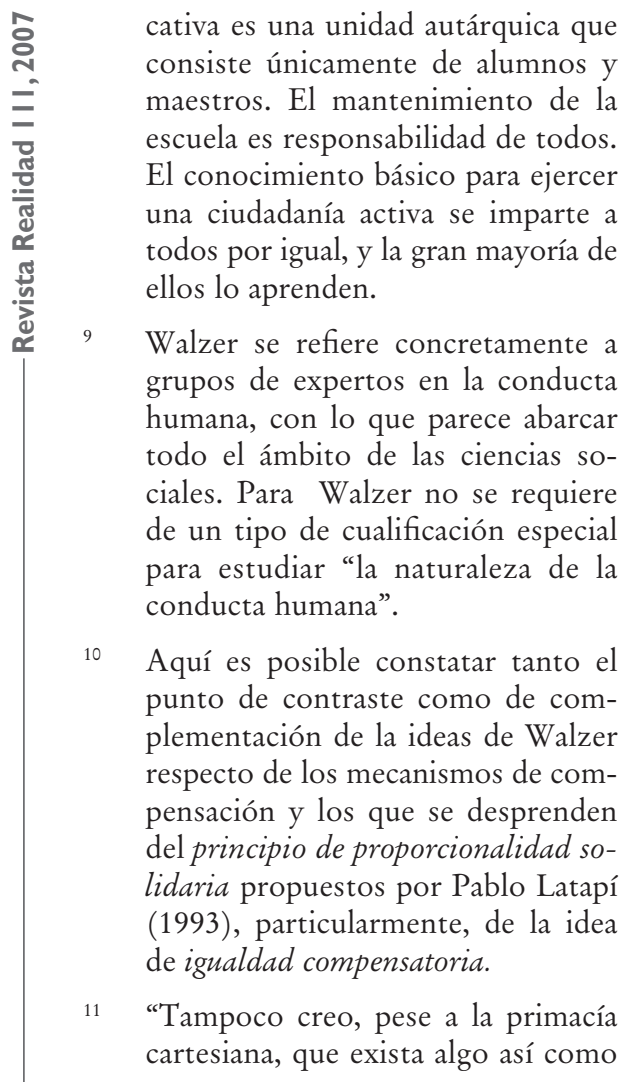

un summum bonum, una jerarquía omniabarcativa de bienes." (Walzer: 1996: 64)

Rawls ha hecho un conjunto significativo de matices a su Teoría de la justicia (1976) - A Theory of Justice (1971, 1a Ed.) que en cierto modo marcan un giro en su pensamiento original, plasmado fundamentalmente en El Liberalismo político (1996).

13 El Frente Zapatista de Liberación Nacional (FZLN) ha sido enfático en este sentido.

14 "La historia nos hace un poco humildes. iAh, la política, qué poco espacio le deja a la historia! Las grandes corrientes de la cultura, que son las que en último término dejan sus huellas, exigen de nosotros una mirada larga, un ritmo más lento de decisiones y una buena dosis de persistencia. No seamos ni tan orgullosos ni tan impacientes. Una política de largo alcance sabe que su trabajo está siempre en los comienzos." Lebret, Louis Joseph, ¿Suicidio o sobrevivencia de Occidente? (1968), p. 267. 